\title{
Accounting Hierarchical Heterogeneity of Rock Massif for Prediction of Mine Seismicity
}

\author{
Olga Hachay¹, Andrey Khachay², Oleg Khachay² \\ ${ }^{1}$ Institute of Geophysics, Ural Branch of Russian Academy of Sciences, Yekaterinburg, Russia \\ ${ }^{2}$ Ural Federal University, Yekaterinburg, Russia \\ Email: olgakhachay@yandex.ru, andrey.khachay@urfu.ru,khachay@yandex.ru
}

How to cite this paper: Hachay, O., Khachay, A. and Khachay, O. (2018) Accounting Hierarchical Heterogeneity of Rock Massif for Prediction of Mine Seismicity. Open Journal of Geology, 8, 187-200. https://doi.org/10.4236/ojg.2018.83012

Received: January 28, 2018

Accepted: March 9, 2018

Published: March 12, 2018

Copyright ( 92018 by authors and Scientific Research Publishing Inc. This work is licensed under the Creative Commons Attribution International License (CC BY 4.0).

http://creativecommons.org/licenses/by/4.0/

(c) (i) Open Access

\begin{abstract}
Purpose: When producing mining operations in high-stress rock massive, technogenic seismicity is manifested. Forecasting and prevention of these events is given much attention in all countries with a developed mining industry. From the point of view of the paradigm of physical mesomechanics, which includes a synergetic approach to changing the state of rock massive of different material composition, this problem can be solved with the help of monitoring methods tuned to the study of hierarchical structural media. Changes in the environment, leading to short-term precursors of dynamic phenomena, are explained within the framework of hierarchical heterogeneity and nonlinearity from observations of wave fields and seismic catalog. For that purpose it is needed to develop new algorithms of modeling wave field propagation through the local objects with hierarchical structure. Design/Methodology/Approach: It had been constructed an algorithm for 3D modeling electromagnetic field for arbitrary type of source of excitation in $\mathrm{N}$-layered medium with a hierarchic conductive and magnetic intrusion, located in the layer number $J$. It had been constructed algorithms for $2 \mathrm{D}$ modeling of sound diffraction and linear polarized transversal seismic wave on an anomaly elastic or dense intrusion of hierarchic structure, located in the layer number $J$ of N-layered elastic medium. We used the method of integral and integral-differential equations for a space frequency presentation of wave field distribution. Findings: From the theory it is obvious that for such complicated medium each wave field contains its own information about the inner structure of the hierarchical inclusion. Therefore it is needed to interpret the monitoring data for each wave field apart, and not mixes the data base. Practical Value/Implications: These results will be the base for constructing new systems of monitoring observations of dynamical geological systems. Especially it is needed to prevent rock shocks in deep mines by their exploitation or natural hazards.
\end{abstract}




\section{Keywords}

Hierarchic Medium, Electromagnetic Field, Seismic Field, Algorithms of Modeling, New Monitoring Systems

\section{Introduction}

The phenomenon of zonal disintegration of rocks around underground excavations was first described in [1]. The formation of structures in irreversible processes is associated with a qualitative jump in reaching threshold (critical) parameters. Self-organization is a supercritical phenomenon when the parameters of the system exceed their critical values. When the system deviates strongly from the equilibrium state, its variables satisfy nonlinear equations. Nonlinearity is an important common feature of processes that go far from equilibrium. In this case, supercritical return of entropy is possible only in the presence of an unusual, special internal structure of the system [2]. This means that self-organization is not a universal property of matter, it exists under certain internal and external conditions and this is not related to a special class of substances. So, there are two classes of irreversible processes: 1) destruction of the structure near the equilibrium position-this is a universal property of systems under arbitrary conditions; 2) the appearance of structures far from the equilibrium position under the conditions that the system is open and has a nonlinear internal dynamics, and its external parameters have supercritical values. I. Prigogine named them as dissipative structures [3]. The study of the morphology and dynamics of migration of these zones is of particular importance in the development of deep-located deposits, complicated by dynamic phenomena in the form of rock impacts. At the same time, geomechanics requires the use of a new model, namely a layered-block model with inclusions of a hierarchical type. In this regard, for the analysis of mechanical fields propagating in such environment, it is necessary to use not kinematic, but dynamic methods based on modeling their propagation through a multi-ranked environment. An important tool for this study is geophysical research. As shown in [4], to describe a geological environment in the form of an array of rocks with its natural and technogenic heterogeneity, we should use its more adequate description, which is a discrete model of the medium in the form of a piecewise heterogeneous block medium with embedded heterogeneities of smaller rank than the block size. This including can be traced several times, i.e. by changing the scale of the investigation; we see that heterogeneities of smaller rank now appear as blocks for heterogeneities of the next rank. Simple averaging of the measured geophysical parameters can lead to changed information about the structure of the medium and its evolution [4].

The Institute of Geophysics of the Ural Branch of the Russian Academy of Sciences has developed a series of devices and interpretation complex for study- 
ing the structure and state of a complex geological environment that has potential instability and the ability to rearrange the hierarchy of the structure due to the significant external impact. The basis of this complex is the developed 3D planshet technique of electromagnetic induction studies in the frequency-geometric version, based on one hand on a software-realized system of interpretation of 3D variable electromagnetic fields [5] [6], and on the other hand on the developed by doctor A.I. Chelovechkov a series of devices for induction studies. At present, the developed methodology is used for mapping and monitoring complex geological environments in the ground and underground (mine) versions. The problems are-to determine the structure of placers (platinum, diamonds), to identify and determine the state of man-made, seasonal and eternal permafrost, to identify zones of disturbance in dams and especially dangerous objects, to solve archaeological problems on the ancient buried precious objects of Russia and Egypt. One of the fundamental problems of mining, which is traditionally attributed to the problems of geomechanics, is the problem of creating a theory and methods for studying the structure and state of rock massifs in order to predict and prevent catastrophic phenomena during the development of deposits [7].

\section{Active Monitoring of the Structure and State of Different Material Content Rock Massif}

Determining the state and dynamics of a rock massif is a more complex problem than mapping its structure. Individual parts of the massif can be in a different stress state, and the corresponding deformations can be either elastic or plastic. The medium can be multiphase. A powerful change in the state of the blocks can lead to stability loss of the whole massif and to a rock shock. The state change is determined both by natural and technogenic impact on the massif and is manifested, among other things, in the form of formation of man-made cavities and pumping mechanical energy during mass explosions provided for mining technologies. The phenomenon of non-stationary state of the rock massif today is a well-known fact [8] [9]. Manifestations of it are in the form of increased permeability due to an increase in the fracturing of rocks, which are already recorded in the form of foreshocks, micro-impacts, rock impacts, rock-tectonic impacts [10]. The latter refer already to catastrophic phenomena, which are initiated by both internal and external technogenic causes. To predict such phenomena during the development of a particular ore deposit, it is important to calibrate the degree of relaxation of the rock mass in response to a controlled mechanical impact. The task includes: -searching and identifying zones-inclusions in a layer-block isotropic by physical properties of the massif and monitoring their migration under the influence of a change in man-made stresses, -evaluation of the state of the massif by the type of inclusions: contact at the boundaries of rocks of different composition, or a fractured medium with varying degrees of moisture saturation and fixation of its change in time. All of the 
above tasks make it urgent to create an integrated approach to the study of the state of the rock massif on the basis of mutually reinforcing geophysical and geomechanical methods. Based on the theoretical analysis of the use of geophysical fields of different nature and new methods for studying rock massive, the main principles of the currently implemented system for monitoring the stressed state of rock massifs, represented by a set of local inclusions having a hierarchical structure, immersed in a layered-block isotropic medium, are formulated. Monitoring is performed to study the nonstationarity of the manifestations of these local zones, related, inter alia, to changes in the state of the massif. The proposed monitoring system is organized in the form of several included cycles: [3] - [2] - [1]. The first cycle (internal) is built on the iterative principle and consists of a sequence of procedures. a) Identification of local zones of heterogeneity in terms of the physical property-electrical conductivity is produced with the help of the method of induction electromagnetic studies developed at the Institute of Geophysics of the Ural Branch of the Russian Academy of Sciences. Further, a geoelectrical model of the studied rock massif is constructed [11] [12]. b) Developing a mathematical model of seismic field with different elastic values [13] [14]. c) Construction a complex electromagnetic and seismic model of the local zone of the rock massif. d) Calculation of the tensor of secondary stresses components created by the zone of heterogeneity in terms of electrical and elastic properties. e) Construction of a unified model for geomechanical and geophysical data of the stressed state within the local zone of heterogeneity. e) The decision to return to a), or the transition to the external stage 2 is determined by the criterion for the fulfillment of point e). In the second cycle, sorting and classification of monitoring application results occurs, the physical and mechanical properties of the studied local zone of the rock massif are analyzed using the approaches [15], the formation of a time series of their changes under the influence of man-made and natural factors. In the third (external) cycle, a quantitative relationship is established between the physical and mechanical parameters that characterize the secondary stress state of each of the local zones of the rock massif, depending on geological, technogenic and seismogenic factors.

At the suggestion of N. P. Vloch, this system was tested on a number of massive, differing in material composition, according to their geological classification (sedimentary, volcanic-sedimentary, and volcanogenic) and in degree of impact hazard. These objects in the Ural were studied: the Magnezitovaya mine, the SUBR, the UUBR, the BKRU-4, the Estyuninsky mine, the Uzelginsky mine; in Siberia, in the Far East: Tashtagol, Nikolaevskij mine (Dalnegorsk). Each of these objects allowed establishing the features of the structure and behavior of the massif, to isolate and rank the factors affecting the stability of the massif. This was possible only with the possibility of comparing various factors within the framework of a single integrated methodology of geophysical and geomechanical research. Consider the results of testing and the effectiveness of the proposed system for monitoring the rock mass at the Magnezitovaya mine (1st 
(internal) cycle). The experience of geophysical work in underground conditions with the use of various physical fields with the purpose of mapping the zones of disturbance of a rock massif and studying the connection of their dynamic manifestations in the specific physical parameters of the massif indicates the highest efficiency and manufacturability of the use of non-contact electromagnetic methods of investigation.

\subsection{Description of Case Sites}

Full-scale experiments were carried out at the Magnezitovaya mine (Satka, Chelyabinsk Region) during the three years 1997-1999, in February-April of each year to study the structure of the interworking space, assess the degree of heterogeneity of the rock block and the variability of its structure and state over time. During the period of providing electromagnetic investigations, the first-stage chambers were working in the process, when the roof and their walls were represented by an array of rocks (dolomite and magnezite), located across the shock of the magnezite deposit. Electromagnetic induction observations were provided at two mutually parallel levels: at the $297 \mathrm{~m}$ horizon in seven mutually parallel excavations (409 - 421) and at the horizon $277 \mathrm{~m}$ in two excavations (211 - 213). At the beginning of the experiment in 1997, they were under-roof workings up to $120 \mathrm{~m}$ length, $3 \mathrm{~m}$ wide and $3 \mathrm{~m}$ height. Then, according to the technology of working out the massif, these workings widened to $8 \mathrm{~m}$ in the part where the magnezite was located. Where there were interlayers of dolomite, the width of the excavations remained the same- $3 \mathrm{~m}$. Then these workings were worked out, their height increased to $10 \mathrm{~m}$, and the inter-working space between the horizons in the process of working the chambers decreased in height from 17 $\mathrm{m}$ to $10 \mathrm{~m}$. The observation profiles passed respectively along the workings practically along their middle. Observations of the modules of the three components of the magnetic field at 5 frequencies (from $5 \mathrm{kHz}$ to $80 \mathrm{kHz}$ ) were carried out with the help of equipment developed at the Institute of Geophysics of the Ural Branch of the Russian Academy of Sciences (the developer A.I. Chevolevochkov) independently for each hole, and the excitation source was in the same hole, as the receiver. One cycle of observations included multifrequency measurements of two horizontal and vertical components of an alternating magnetic field when the receiver moved with a given step and fixed source position, then the source moved to another point of the profile and the measurement cycle was repeated. In the present experiment, four cycles of observations were made in all the excavations.

\subsection{Analysis of Electromagnetic Monitoring Data}

After appropriate processing of the data within the framework of a specialized system, developed by us, the distribution of effective resistance as a function of distance was obtained for all frequencies. Data analysis was carried out within the framework of the concept of three-stage interpretation. Thus, using the algo- 
rithms for the interpretation of the first stage [12], one-dimensional models of the distribution of the resistivity in the interworking space were obtained, which for all workings correspond to the model of a homogeneous half-space with a resistance of $1000 \mathrm{ohms}$. From the analysis of the experimental values of the effective resistivity in the chambers of the horizon, $297 \mathrm{~m}(\mathrm{a}, \mathrm{b})$ and $277 \mathrm{~m}$ (in) before and after the chambers working (frequency $40 \mathrm{kHz}$ ), after preliminary filtration treatment [12] it follows that the geoelectrical parameter is eff., characterizing the resistivity of the rock massif, as a host environment, does not change over time (data obtained in 1997 and 1998). Intensive man-made influence on the eff. with increased tremor as a result of blasting operations during field development is practically not manifested.In [16] for the construction of a volume model for the distribution of local geoelectrical heterogeneities, a geoelectrical heterogeneity parameter $\delta$ was introduced as a function of the frequency, the distance between the point of reception and the location of the source and the distribution of the resistivity, both in the host environment and inside the local zones, which turned out to be an important stress-sensitive parameter. We analyzed the average geoelectrical heterogeneity parameter determined for each collocation for each frequency separately in the form (1)-(2):

$$
\begin{aligned}
& \bar{\delta}_{m}^{i}=\frac{1}{K} \sum_{k=1}^{K} \delta_{m k}^{i} ; \\
& \delta_{m k}^{i}=\frac{\left|H_{\phi m}^{i}\right|}{\left|H_{\rho m k}^{i}\right|} \times 100 \%
\end{aligned}
$$

$i$-number of frequency $(1-5 \mathrm{kHz}, 2-10 \mathrm{kHz}, 3-20 \mathrm{kHz}, 4-40 \mathrm{kHz}, 5-80$ $\mathrm{kHz}$ ); $K$-the number of movements of the excitation source within the observation section; $k$ is the location number of the source of excitation; $m$ is the location number of the receiver.

The analysis of the results obtained in 1997, 1998 full-scale experiments shows that the effect of changing the geoelectrical heterogeneity parameter over time is significant and fixed in the framework of the observations, and as a rule these changes are larger in absolute magnitude at a frequency of $5 \mathrm{kHz}$, compared to with the remaining frequencies. To interpret these data, we created an iterative algorithm based on the minimization of the functional, taking into account the mutual lateral influence and the influence of "top-bottom" of the following kind (3):

$$
Q^{i}=\frac{1}{N} \sum_{N} \frac{1}{M_{N}} \sum_{m=1}^{M_{N}}\left|\bar{\delta}_{m}^{i}-\bar{\delta}_{m}^{T i}\right| ;
$$

where $N$-is the number of considered interactions in the profile data of the volume observation system, $M_{N}$-the number of observation points on each observation profile, $\bar{\delta}_{m}^{T i}$-the approximation design or the theoretical average parameter of the geoelectrical heterogeneity at the observation point of the number $m$ [12]. As a result of minimization, the following parameters are determined: $n$ is the number of anomalous objects, the coordinates of the left end of each 
source and its length, the vertical coordinate, and $M$ is the moment of the source whose value is proportional to the conductivity of the object creating in the magnetic field an effect equivalent to the field of singular sources. A volume model for the distribution of local heterogeneous zones was constructed as a result of the interpretation of the data of electromagnetic studies of 1998 at two levels: the horizon of $297 \mathrm{~m}$ and $277 \mathrm{~m}$ [11]. A similar model was constructed according to 1997 data. For one cycle of observations, the position of the sources associated with the anomalous zones, determined from data for different frequencies, does not change, but the value $M$ depends on the frequency. For different observation cycles, the geometric location of the anomalous zones also changes, indicating the reaction of the massif to the technogenic effect. Prior to the testing of the chambers, the sources of the anomalous field were located exclusively in the interworking space of the horizons $297 \mathrm{~m}$ and $277 \mathrm{~m}$. The zonality of their depth distribution was revealed: depths of $2-3 \mathrm{~m}, 4.5-6 \mathrm{~m}, 8.5-9$ $\mathrm{m}$, and $10-13.5 \mathrm{~m}$ from the soil of the 400 chambers were identified. After working off, these zones also exist in the roof of the chambers of the horizon 297 m. Analysis of the average parameter $M$ for the frequency showed that for most anomalous zones its value is less than 0.2 , which is assumed to be the background one. Several anomalous zones have been identified, for which $M>0.2$. It is known that if the anomalous zone is associated with the presence of a contact of two media of different conductivity, then the value of the moment of the singular source should not depend on the frequency. In the case of fractured media, the dependence of the moment or resistivity in the anomalous zone on the frequency can be observed, and the more the moisture saturation of the medium, the greater the variance value can be expected. Analysis of the dispersion of the parameter $M$ as a function of frequency for the most important anomalous identified zones showed that the highest dispersion values occur at frequencies of 5 and $10 \mathrm{kHz}$. This effect makes it possible to identify weakened fractured zones, separating them from ordinary undisturbed contacts and inclusions of other rocks. There is a qualitative morphological similarity of the dispersion curves before and after the technogenic impact on the massif, while the variance in this case decreased after the testing of the chambers.

Geomechanical studies were carried out to test the hypothesis about the nature of anomalous geoelectrical zones associated with excessive fracturing. As a result of the comparison of the results obtained, the following features were revealed: according to the data of geomechanical studies, in the second and third wells, zones of intense fracturing related to the fracture zones were found in the first well of such zones were not found. Electromagnetic studies were carried out earlier than geomechanical studies and, based on the results of their interpretation; a predicted section was constructed, including the position of the inhomogeneous zones [11]. Geomechanical studies were carried out after the 417 chambers were worked out, while 217 remained underlying hole. The location of the fracture zone in the second well coincides with the predicted position of the 
fracture zone by electromagnetic data. The first well went past the fracture zone and this also corresponds to the prognosis section, constructed according to electromagnetic data. The third well passed through the zone of disturbance, but according to the electromagnetic data, before the 417 chamber was worked out, it was closer to the 417 chamber soil. It is likely that after she worked, she moved down. In works [17] [18] [19], using 3D electromagnetic induction spatial-temporal monitoring, it was possible to show that the model of a hierarchical discrete medium is applicable for describing the structure of an array of rocks of different material composition [20]. Within the framework of a specific modification of the method, it was possible to trace two hierarchical levels.

\section{Study of the Stress-Deformed State of Block Layered Medium with Hierarchical Inclusions}

To solve the problems of geological and geophysical mapping, a model of a layered-block medium with homogeneous and inhomogeneous inclusions in physical properties is currently widely used, within the framework of which apparatus-methodological complexes for studying three-dimensional inhomogeneous media with the corresponding interpretation theory of geophysical data have been created [21]. When studying spatial-temporal changes in the structure, physical properties of a geological environment or an array of rocks and the stress-strain or phase state associated with them, the model of a stratified-block medium with inclusions becomes more complicated: it is a two rank chain in a general hierarchically inhomogeneous model of the medium. The model of a hierarchically heterogeneous medium for describing the processes of deformation and destruction of the geophysical environment was first proposed by Academician M.A. Sadovskiy [4]. The development and use of the hierarchical block model of the environment at a qualitative level is devoted to a number of works by the staff of the Institute of Physics of the Russian Academy of Sciences [22] [23]. Theoretical and experimental results obtained on samples [24], with the help of which an approach based on the concept of dissipative structures in no equilibrium systems, is important for understanding the formation and development of the hierarchy of structural levels of deformation in solids [25]. In the works [26] [27] NA. Karaev summarized the results of seismic studies on the allocation of sections of the earth's crust with the structure of a heterogeneous type. Heterogeneity, in the author's opinion, is the most important feature of rocks, caused by the uneven distribution of geological irregularities in the space in the form of inclusions of all scales, i.e. in fact, the study of the structure and dynamics of heterogeneous sections of the earth's crust is necessary using the concepts of hierarchical models. The phenomena of zonal disintegration of rocks around underground excavations, related to the discreteness and fragmentation of the medium, are described by academician E. I. Shemjakin with co-authors [1] and obtained the discovery status [28]. These phenomena occur both in shallow mines (up to $500 \mathrm{~m}$ ), and in deep mines (more than $500 \mathrm{~m}$ ). The results 
of geological and geophysical studies of super deep wells show that with increasing depth, the complexity of the geological structure of the medium does not decrease. Finally, the problem of monitoring and forecasting the state of the geological environment requires careful selection from existing research techniques for three-dimensional environments that allow space-time (frequency) scaling and focusing. Our work is devoted to the development of an algorithm for modeling the electromagnetic and seismic effect within the framework of the frequency-geometric technique when investigating a conductive or elastic inclusion with a hierarchical structure immersed in a horizontally layered medium. The algorithm is constructed using an iterative approach to solving volumetric integral equations for the alternating electromagnetic field in the frequency domain. For the model: the conducting inclusion located in an arbitrary layer of a five-layer medium, when the electromagnetic field is excited by a plane wave, a vertical magnetic or horizontal electric dipole, is described in [29].

\subsection{An Algorithm for Modeling the Propagation of an Alternating Electromagnetic Field in an N-Layer Medium with a Conducting Inclusion of a Hierarchical Type Located in the J-th Layer}

Let the local heterogeneity has the following structure: at the first hierarchical level, this is the heterogeneity of the volume $\mathrm{V}_{1}$ with the conductivity $\sigma_{a 1}$; at the second level, these are the heterogeneities located inside the volume $\mathrm{V}_{1}$ and occupying the volume $V_{2}$ with the conductivity $\sigma_{a / 2}$ in the general case. We will consider the simpler case, when heterogeneities of the second and higher rank will have the same conductivity within their rank, that is, $\sigma_{a 2}$. The heterogeneities of the third rank will occupy the volume $V_{3}$ within the volume $V_{2}$ with the conductivity $\sigma_{a 3}$, etc. The parameters of the host horizontally layered medium have the form $\left\{\sigma_{k}, h_{k}\right\}$, where $k=1, \cdots, N, h_{k}$ are the total thicknesses of the layers, $i$ is the rank or hierarchical level number. The problem is considered for a magneto-homogeneous medium in the quasistationary approximation.

The volume integral equations and, respectively, integral representations for the electric and magnetic field components are written out as:

$$
\begin{aligned}
& \boldsymbol{E}_{i}\left(M_{0}\right)=\boldsymbol{E}_{0}^{i-1}\left(M_{0}\right)+\left(\sigma_{a i}-\sigma_{k\left(M_{0}\right)}\right) \int_{V_{i}} \boldsymbol{E}_{i}(M) \hat{G}_{E}\left(M, M_{0}\right) \mathrm{d} V_{i} \\
& \boldsymbol{H}_{i}\left(M_{0}\right)=\boldsymbol{H}_{0}^{i-1}\left(M_{0}\right)+\frac{\left(\sigma_{a i}-\sigma_{k\left(M_{0}\right)}\right)}{i \omega \mu_{0}} \int_{V_{i}} \boldsymbol{H}_{i}(M) \hat{G}_{H}\left(M, M_{0}\right) \mathrm{d} V_{i}
\end{aligned}
$$

where, $i$ is the number of iterations, and $\hat{G}_{E}\left(M, M_{0}\right)$ and $\hat{G}_{H}\left(M, M_{0}\right)$ are the Green's tensors of the layered medium, which are determined by the known method described in [6]. For $i=1$, and are the components of the electric and magnetic exciting or normal field intensities of the layered medium. When $i=$ $2, \cdots L$ and are the components of the strengths of the electric and magnetic fields of the previous rank, taking into account the heterogeneities of the pre- 
vious rank, calculated from formulas (4) and (5). The distribution of the electric and magnetic fields can be analyzed sequentially at each iteration step before switching on the heterogeneities of the new hierarchical level.

\subsection{Algorithm for Modeling the Diffraction of Sound on a Two-Dimensional Elastic Heterogeneity of a Hierarchical Type Located in the J-th Layer of an N-Layer Medium}

An algorithm for simulating the diffraction of sound on a two-dimensional elastic homogeneous inclusion located in the J-layer of an N-layer medium is written in [30].

$$
\begin{aligned}
& \frac{\left(k_{1 j i}^{2}-k_{1 j}^{2}\right)}{2 \pi} \iint_{S c} \varphi(M) G_{S p, j}\left(M, M^{0}\right) \mathrm{d} \tau_{M}+\frac{\sigma_{j a}}{\sigma_{j i}} \varphi^{0}\left(M^{0}\right) \\
& -\frac{\left(\sigma_{j a}-\sigma_{j i}\right)}{\sigma_{j i} 2 \pi} \oint_{C} G_{S p, j} \frac{\partial \varphi}{\partial n} \mathrm{~d} c=\varphi\left(M^{0}\right), M^{0} \in S_{C} \\
& \frac{\sigma_{j i}\left(k_{1 j i}^{2}-k_{1 j}^{2}\right)}{\sigma\left(M^{0}\right) 2 \pi} \iint_{S c} \varphi(M) G_{S p, j}\left(M, M^{0}\right) \mathrm{d} \tau_{M}+\varphi^{0}\left(M^{0}\right) \\
& -\frac{\left(\sigma_{j a}-\sigma_{j i}\right)}{\sigma\left(M^{0}\right) 2 \pi} \oint_{C} G_{S p, j} \frac{\partial \varphi}{\partial n} \mathrm{~d} c=\varphi\left(M^{0}\right), M^{0} \in S_{C}
\end{aligned}
$$

where $G_{S p, j}\left(M, M^{0}\right)$ is the source function of the seismic field of the problem under consideration; $k_{1 j i}^{2}=\omega^{2}\left(\sigma_{j i} / \lambda_{j i}\right)$; is the wave number for the longitudinal wave, in the above expression, the index $j i$ denotes the belonging of the medium properties inside the heterogeneity, ja-outside the heterogeneity, the Lamé constant, $\sigma$-the density of the medium, $\omega$-circular frequency, -potential of a normal seismic field in a layered medium in the absence of an heterogeneity: $\varphi_{j i}^{0}=\varphi_{j}^{0}$. The idea presented in the previous paragraph for the electromagnetic field is also realized for a seismic field for a two-dimensional case of propagation of a longitudinal wave through a local heterogeneity with a hierarchical structure located in the $j$-th layer of the $N$-layered medium.

$$
\begin{aligned}
& \frac{\left(k_{1 j i}^{2}-k_{1 j}^{2}\right)}{2 \pi} \iint_{S c l} \varphi_{l}(M) G_{S p, j}\left(M, M^{0}\right) \mathrm{d} \tau_{M}+\frac{\sigma_{j a}}{\sigma_{j i}} \varphi_{l-1}^{0}\left(M^{0}\right) \\
& -\frac{\left(\sigma_{j a}-\sigma_{j i l}\right)}{\sigma_{j i l} 2 \pi} \oint_{C l} G_{S p, j} \frac{\partial \varphi_{l}}{\partial n} \mathrm{~d} c=\varphi\left(M^{0}\right), M^{0} \in S_{C l} \\
& \frac{\sigma_{j i} l\left(k_{1 j i l}^{2}-k_{1 j}^{2}\right)}{\sigma\left(M^{0}\right) 2 \pi} \iint_{S c l} \varphi_{l}(M) G_{S p, j}\left(M, M^{0}\right) \mathrm{d} \tau_{M}+\varphi_{l-1}^{0}\left(M^{0}\right) \\
& -\frac{\left(\sigma_{j a}-\sigma_{j i} l\right)}{\sigma\left(M^{0}\right) 2 \pi} \oint_{C l} G_{S p, j} \frac{\partial \varphi_{l}}{\partial n} \mathrm{~d} c=\varphi_{l}\left(M^{0}\right), M^{0} \in S_{C l}
\end{aligned}
$$

$G_{S p, j}\left(M, M^{0}\right)$-function of the source of the seismic field of the problem under consideration, it coincides with the function of expression (64). $k_{1 j i l}^{2}=\omega^{2}\left(\sigma_{j i l} / \lambda_{j i l}\right)$ - the wave number for the longitudinal wave, in the above expression, the index 
$j i$ denotes the property of the medium inside the heterogeneity, $j a$-outside the heterogeneity, the index $I=1, \cdots L$-the number of the hierarchical level, $\mathbf{u}_{l}=\operatorname{grad} \varphi_{l}, \varphi_{l-1}^{0}$-the potential of the normal seismic field in the layered medium in the absence of heterogeneity of the previous rank, if $l=2, \cdots \mathrm{L}$, $\varphi_{l-1}^{0}=\varphi_{l}$.

If, moving to the next hierarchical level, the two-dimensionality axis does not change, and only the geometry of sections of embedded structures changes, then it is possible to write out an iterative process of modeling the seismic field similarly $(4,5)$ (the case of the formation of only a longitudinal wave). The iterative process refers to modeling the displacement vector from the previous hierarchical level to the next level. Within each hierarchical level, the integro-differential equation and the integro-differential representation are computed using algorithms (7). If at some hierarchical level the structure of the local heterogeneity breaks down into several heterogeneities, then the double and surface integrals in expressions (7) are taken over all heterogeneities. In this algorithm, the case is considered when the physical properties of the heterogeneities of the same level are identical, only the boundaries of the regions differ.

\subsection{Algorithm for Modeling Diffraction of a Linearly Polarized Transversal Wave on a Two-Dimensional Elastic Heterogeneity of a Hierarchical Type Located in the J-th Layer of an N-Layer Medium}

Similarly to (7), the same process is written for modeling the propagation of an elastic transverse wave in an n-layer medium with a two-dimensional hierarchical structure of arbitrary cross-section morphology using integral relations [30]. $G_{S s, j}\left(M, M^{0}\right)$-the function of the source of the seismic field of the problem under consideration, $k_{2 j i l}^{2}=\omega^{2}\left(\sigma_{j i l} / \mu_{j i l}\right) ; \mu$ is the Lamé constant.

$$
\begin{aligned}
& \frac{\left(k_{2 j i}^{2}-k_{2 j}^{2}\right)}{2 \pi} \iint_{S C l_{l}} u_{x l}(M) G_{S s, j}\left(M, M^{0}\right) \mathrm{d} \tau_{M}+\frac{\mu_{j a}}{\mu_{j i l}} u_{x(l-1)}^{0}\left(M^{0}\right) \\
& +\frac{\left(\mu_{j a}-\mu_{j i l}\right)}{\mu_{j i l} 2 \pi} \oint_{C l} u_{x l}(M) \frac{\partial G_{S s, j}}{\partial n} \mathrm{~d} c=u_{x l}\left(M^{0}\right), M^{0} \in S_{C l} \\
& \frac{\mu_{j i l}\left(k_{2 j i l}^{2}-k_{2 j}^{2}\right)}{\mu\left(M^{0}\right) 2 \pi} \iint_{S C l_{l}} u_{x l}(M) G_{S s, j}\left(M, M^{0}\right) \mathrm{d} \tau_{M}+u_{x(l-1)}^{0}\left(M^{0}\right) \\
& +\frac{\left(\mu_{j a}-\mu_{j i l}\right)}{\mu\left(M^{0}\right) 2 \pi} \oint_{C l} u_{x l}(M) \frac{\partial G_{S s, j}}{\partial n} \mathrm{~d} c=u_{x l}\left(M^{0}\right), M^{0} \in S_{C l}
\end{aligned}
$$

Thus, the iterative processes (7) and (8) make it possible to determine, for given elasticity modules, the layered medium that encloses the hierarchical heterogeneity and in the heterogeneity at each hierarchical level analyze the spatial distribution of the components of the seismic field. Then, using the known formulas [31] for each hierarchical level, calculate the distribution of the components of the strain tensor and the stress tensor from the distribution of the dis- 
placement components. This information plays an important role in assessing the state of the environment, depending on the structure of its hierarchy and the degree of its variability.

\section{Conclusions}

The results of active electromagnetic induction monitoring in the shock proof mine of the Tashtagolsky mine allow the following conclusions to be drawn [20]:

- massif of rocks represents a multi-ranked hierarchical structure; the study of the dynamics of a state and its structure can be conducted only with the help of geophysical methods tuned to such a model of the environment.

- the use of a multi-level induction electromagnetic method with a controlled source and a corresponding processing and interpretation technique allowed us to trace two hierarchical levels and identify the zones of disintegration that are an indicator of the stability of the array.

- zones of disintegration in the circumscribed area are asymmetrical in soil and roof and discrete: i.e. there are intervals in the near-service space for their complete absence. The maximum changes in the array under the technogenic influence occur precisely in the morphology of the spatial position of these zones as a function of time.

- the introduction of a new integral parameter of the intermittent intensity distribution of the disintegration zones makes it possible to proceed to a detailed classification of the array by the degree of stability and introduce quantitative criteria for this array [20].

- introduction of the proposed integrated passive and active geophysical monitoring aimed at studying the transient processes of redistribution of the stress-strain and phase states, contributes to the prevention of catastrophic dynamic manifestations during the development of deep-located deposits.

At present, theoretical results on the modeling of the electromagnetic and seismic field in a layered medium with inclusions of a hierarchical structure are claimed [32]. Algorithms for modeling in the electromagnetic case for 3D heterogeneity are constructed, in the seismic case for 2D heterogeneity [30] [33] [34] [35].

It is shown that with increasing degree of hierarchy of the medium, the degree of spatial nonlinearity of the distribution of the components of the seismic and electromagnetic fields increases, which corresponds to the detailed monitoring experiments conducted in the shock-hazard mines of the Tashtagolsky mine and the SUBR. The constructed theory demonstrated how the process of integrating methods that use the electromagnetic and seismic field to study the response of a medium with a hierarchical structure becomes more complicated.

\section{References}

[1] Shemjakin, E.I., et al. (1986) The Effect of Zonal Disintegration of Rocks around Underground Excavations. DAN USSR, 289, 1088-1094. 
[2] Ebeling, V. (1979) Formation of Structures in Irreversible Processes. Mir, Moscow.

[3] Glensdorf, P. and Prigogine, I. (1973) Thermodynamic Theory of Structure, Stability and Fluctuations. The World, Moscow.

[4] Sadovskiy, M.A., et al. (1987) Deformation of the Geophysical Environment and Seismic Process. Nauka, Moscow.

[5] Hachay, O.A. (2000) Complex Geophysical Studies (Theory and Practical Results). Ural s Geophysical Bulletin. 1, 107-110.

[6] Hachay, O.A. (2003) To the Question of the Study of the Structure, the State of the Geological Heterogeneous Environment, and Their Dynamics within the Framework of a Discrete and Hierarchical Model. Geomechanics in Mining, IGD UB RAS, Ekaterinburg, 30-38.

[7] Vloch, N.P. (1994) Management of Mining Pressure in Underground Mines. Nedra, Moscow.

[8] Adushkin, A.A. and Tsvetkov, V.M. (1997) Influence of Structure and Geodynamics on the Stress State of the Earth's Crust. Conference on Problems of Rocks Mechanics, St. Petersburg, 7-12.

[9] Shkuratnik, V.L. and Lavrov, A.V. (1997) Effects of Memory in Rocks. Physical Regularities, Theoretical Models. Publishing House of the Academy of Mining Sciences, Moscow.

[10] Catalog of Rock Impacts on Ore and Non-Ore Deposits (1986) VNIMI, Leningrad.

[11] Hachay, O.A. and Khachay, O.Y. (2017) New Monitoring Systems to Substantiate Mine Seismicity, Taking into Account the Hierarchical Heterogeneity of the Rock Massive Monitoring. Science and Technology, 1, 26-33.

[12] Hachay, O.A. and Novgorodova, E.N. (1999) Use of a New Three-Dimensional Method of Electromagnetic Studies of the Structure of Mountain Ranges. Physics of the Earth, 5, 61-79.

[13] Hachay, O.A. and Khachay, M.Y. (2003) New Approaches to the Analysis of Complex Nonlinear Nonstationary Media. Ural s Geophysical Bulletin, 5, 98-105.

[14] Hachay, O.A. and Khinkina, T.A. (1998) Mathematical Modeling of the Stress State Change during Mining of the Rock Massive by the Chamber Method. Problems of Geotechnology and Non-Natural Sciences, V.1. Ekaterinburg, 217-225.

[15] Vloch, N.P. (1995) Geomechanical Support of Effective and Safe Development of Ore Deposits by Underground Method. Gorniy Vestnik, 4, 28-31.

[16] Hachay, O.A., Novgorodova, E.N. and Khachay, O.Y. (2003) A New Technique for Detecting Zones of Disintegration in the Near-Development Space of Rock Massifs of Different Material Composition. Mining Information and Analytical Bulletin, 11, 26-29.

[17] Hachay, O.A. (2004) On the Study of the Structure and State of a Geological Heterogeneous Nonstationary Medium in the Framework of a Discrete Hierarchical Model. Russian Geophysical Journal, 33-34, 32-37.

[18] Hachay, O.A., Novgorodova, E.N. and Khachay, O.Y. (1998) Three-Dimensional Electromagnetic Studies of the Structure and State of an Array of Rocks in Mining Geophysics. Proceedings of the International Conference. St. Petersburg, 22-25 June 1998, 591-598.

[19] Hachay, O.A., Khinkina, T.A. and Khachay, O.Y.(2002) Reflection of the Dynamics of the Phase State of an Array of Rocks Based on the Results of Multi-Level Electromagnetic Monitoring Studies in a Shock-Hazard Mine (Tashtagol). Mining Information and Analytical Bulletin, 11, 109-114. 
[20] Hachay, O.A. (2007) Investigation of the Development of Instability in an Array of Rocks Using the Method of Active Electromagnetic Monitoring. Physics of the Earth, 4, 65-70.

[21] EAGO (2000) 300 Years of Mining and Geological Service in Russia. Abstracts of the Reports of the International Geophysical Conference, EAGO, Moscow.

[22] (1989) Discrete Properties of a Geophysical Environment. Nauka, Moscow.

[23] Rodionov, V.N. (1989) On the Modeling of Natural Objects in Geomechanics. Nauka, Moscow.

[24] Panin, V.E. (1985) Structural Levels of Deformation of Solids. SB AS USSR, Nauka, Novosibirsk.

[25] Nikolis, G. (1979) Self-Organization in No Equilibrium Systems. Mir, Moscow.

[26] Karaev, N.A. (2000) Ore Seismic Prospecting. CJSC. Geoinformmark, Moscow.

[27] Karaev, N.A. (2000) Seismic Heterogeneity of the Earth's Crust and the Problems of Interpreting the Results of Regional Observations in the "Near" Zone. In: No Classical Geophysics, Abstracts of Presentations of International Conference, $28 \mathrm{Au}$ gust-1 September 2000, Saratov, 30-32.

[28] Shemjakin, E.I., Kurlenja, M.V., Oparin, V.N., et al. (1992) The Phenomenon of Zonal Disintegration of Rocks around Underground Excavations. Bulletin of Discoveries, $1,39$.

[29] Hachay, O.A. (1994) Mathematical Modeling and Interpretation of Alternating Electromagnetic Field in the Inhomogeneous Crust and upper Mantle of the Earth. Thesis of Doctor of Physical and Mathematical Sciences, Institute of Geophysics UB RAS, Sverdlovsk.

[30] Hachay, O.A. and Khachay, A.Y. (2011) On the Integration of Seismic and Electromagnetic Active Methods for Mapping and Monitoring the State of Two-Dimensional Heterogeneities in an N-Layer Medium. Bulletin of South Ural State University, Series Computer Technologies, Control, Radio Electronics, 13, 49-56.

[31] Lurie, A.I. (1956) Spatial Problems in the Theory of Elasticity. Gos. Publishing House of Technical and Theoretical Literature, Moscow.

[32] Hachay, O.A. and Khachay, A.Y. (2012) The Study of the Stress-Deformation State of Hierarchical Media. Vol. 1, IPHZ RAS, Moscow, 114-117.

[33] Hachay, O.A. and Khachay, A.Y. (2016b) Modeling the Propagation of a Seismic Field in a Layered-Block Elastic Medium with Hierarchical Plastic Inclusions. Mining Information and Analytical Bulletin, 12, 318-326.

[34] Hachay, O.A., Khachay, O.Y. and Khachay, A.Y. (2015) New Methods of Geoinformatics Monitoring of Wave Fields in Hierarchical Environments. Geoinformatics, 3, 45-51.

[35] Hachay, O.A., Khachay, O.Y. and Khachay, A.Y. (2016). New Methods of Geoinformatics for the Integration of Seismic and Gravitational Fields in Hierarchical Environments. Geoinformatics, 3, 25-29. 